\title{
Cartesian Impedance Control on Five-Finger Dexterous Robot Hand DLR-HIT II with Flexible Joint
}

\author{
Zhaopeng Chen ${ }^{1,2}$, Neal Y. Lii ${ }^{1}$, Minghe $\mathrm{Jin}^{2}$, Shaowei Fan ${ }^{2}$, and Hong Liu ${ }^{1,2}$ \\ 1 Institute of Robotics and Mechatronics, German Aerospace Center, DLR, 82234 \\ Wessling, Germany \\ 2 State Key Laboratory of Robotics and System, Harbin Institute of Technology, \\ HIT, 150001 Harbin, China \\ zhaopeng. chen@dlr.de
}

\begin{abstract}
This paper presents an impedance controller for five-finger dexterous robot hand DLR-HIT II, which is derived in Cartesian space. By considering flexibility in finger joints and strong mechanical couplings in differential gear-box, modeling and control of the robot hand are described in this paper. The model-based friction estimation and velocity observer are carried out with an extended Kalman filter, which is implemented with parameters estimated by Least Squares Method. The designed estimator demonstrates good prediction performance, as shown in the experimental results. Stability analysis of the proposed impedance controller is carried out and described in this paper. Impedance control experiments are conducted with the five-finger dexterous robot hand DLR-HIT II in Cartesian coordinates system to help study the effectiveness of the proposed controller with friction compensation and hardware architecture.
\end{abstract}

Keywords: dexterous robot hand, flexible joint, Cartesian space, impedance control, friction compensation.

\section{Introduction}

Since intelligence and hands are the two features that most distinguish humans from other animals, researchers have long been fascinated by the mechanical dexterity of the human hand. Nevertheless it is still an unmatched standard if a broad scope of manipulation tasks is considered [1. It is highly anticipated that the forthcoming anthropomorphic robot hand will eventually supplant human labor in intricate and hazardous tasks execution.

Many multifingered robot hands have been developed mainly in two categories: With actuators mounted in the robot hand, or located outside the robot hand and power transmitted though tendon cables. The latter robot hands (e.g., Stanford-JPL hand by Salisbury et al. 2], the Utah/MIT hand by Jacobsen et al. 3], and the robonaut hand by Lovchik et al. 4]) suffer from the elasticity of the tendon cable with inaccurate joint angle control, and are problematic in 
commercial manufacturing due to their mechanical complexity. However the former group of robot hands (e.g., the Gifu Hand by Kawasaki et al. [5], the DLR Hand II by Butterfass [6] and the DLR/HIT Hand I by Liu [7]) face other problems, such as insufficient fingers and joints, inappropriate large size and weight for practical task.

Manipulative dexterity and grasp robustness are two main types of functional requirements for a robot hand. By any reasonable definition, manipulation fundamentally requires mechanical interaction with the object being manipulated. The problems of controlling the mechanical interaction between a manipulator and its environment have been addressed by many researchers [8] [9] [10]. Hogan introduced a framework for impedance control [11, which can be used to achieve compliant manipulation and reliable grasping with different objects in unknown environments. Extensive literature exists on theories and application of impedance control law. Kazerooni et al. [12] proposed practical compliant impedance control law in Cartesian coordinates frame, by considering manipulator and environment as a whole system to preserve the global stability. A unified approach for motion and force control of robot manipulator in operational space with kinematic singularity treatment was designed by Khatib [13. An internal force-based impedance control scheme for cooperating manipulators was introduced by Bonitz and Hisa [14]. Asymptotic stability was proven using Lyapunov theory and simulation results were presented to validate the proposed concepts.

The above approaches of impedance control were developed for robot systems with rigid body models, which neglect effects of joint elasticity. However, joint flexibility is non-neglectable in robots powered by DC or AC motor in series with harmonic drivers, which are common in many precision positioning and light weight applications. An effective Cartesian impedance control method based on singular perturbation model was proposed by Spong [15, in which the flexibility of joints is treated in a sufficiently fast inner torque control loop. Other decoupling-based approaches are given by Lin and Goldenberg [16, De Luca [17, which provide a linearized closed loop system, and ensure global asymptotic stability. However, these approaches resulted in moderate performance and robustness in joint torque sensor-based robots, due to the requirement on high derivative of link side positions and accurate robot model. To achieve both theoretically sound and practically feasible impedance controller for robots with elastic joints, Albu-schaeffer and Ott et al. [18] 19] introduced passivity-based impedance control which solely relies on motor position, joint torque, and their first-order derivatives. However, nonlinear effects such as friction compensation of the robots with elastic joint are not completely implemented in the above work. In this paper, a impedance control strategy for dexterous robot hand is proposed with friction compensation, which is estimated by extended Kalman filter.

This paper is organized as follows: Section 2 presents the dexterous robot hand system and robot model; Section 3 presents the joint and cartesian impedance controller of the dexterous robot hand, global asymptotic stability is also proven; Section 4 deals with the velocity observer and friction compensation based on 
extended Kalman filter; experimental results are presented in Section 5. Finally, the conclusions of this work are drawn and presented in Section 6 .

\section{Robot Hand System and Dynamic Model}

By considering the requirements for dexterous grasping and manipulation of objects, A five-fingered dexterous robot hand DLR-HIT II was developed jointly by German Aerospace Center, Germany and Harbin Institute of Technology, Chian [20, as illustrated in Fig. 1]. The proposed hardware and software control architecture is composed of multisensory system, three DSP/FPGA based processing units for the distal controller, finger controller and palm controller, external real-time PC and multipoint real-time communication system.

To realize an anthropomorphic robot hand design, as well as augmentation of available torque for a given motor size and a reduced weight, the proximal joint of the robot finger is realized as coupled joints using a differential gear with a harmonic drive and timing belt. As shown in Fig. 2, the differential gear box is composed of four conical gears. Movement of one joint is realized by the coordinated movement of two actuators. Without considering the elasticity, the transformation matrix for each finger post differential gearing can be written as:

$$
\begin{aligned}
\boldsymbol{\theta}_{l} & =\boldsymbol{L} \boldsymbol{\theta}_{\boldsymbol{q}} \\
\tau & =\boldsymbol{L}^{T} \tau_{l}
\end{aligned}
$$

with

$$
\boldsymbol{L}=\left[\begin{array}{ccc}
\mathbf{1} & \mathbf{0} & \mathbf{0} \\
\mathbf{0} & \mathbf{1} & -\mathbf{1} \\
\mathbf{0} & \mathbf{1} & \mathbf{1}
\end{array}\right] \in \mathbb{R}^{3 \times 3}
$$

$\boldsymbol{\theta}_{\boldsymbol{l}}$ and $\boldsymbol{\theta}_{\boldsymbol{q}}$ denote the link side position and joint position expressed in joint coordinates, respectively. $\boldsymbol{q}$ represents $\boldsymbol{\theta}_{\boldsymbol{q}}$ later in this article to indicate the
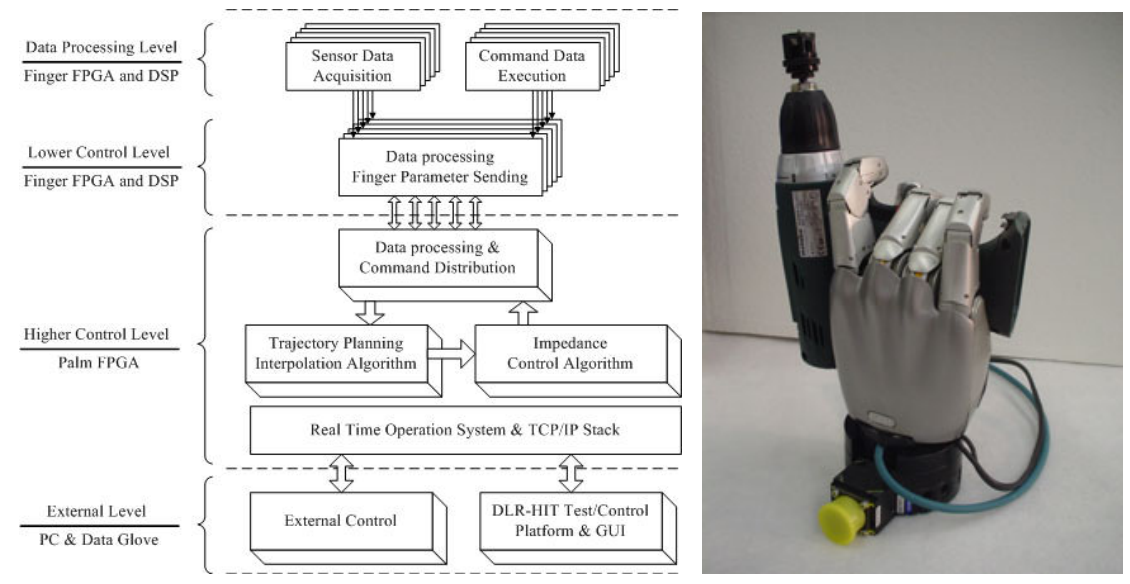

Fig. 1. Dexterous Robot Hand DLR-HIT II 


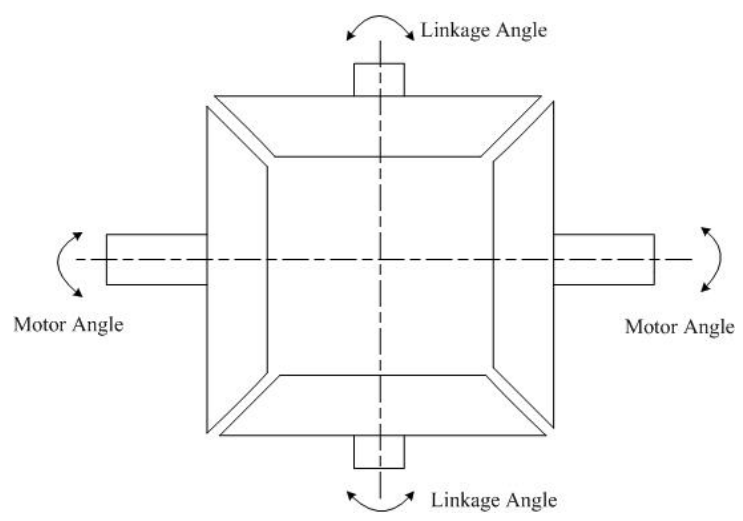

Fig. 2. Modeling of Differential Bevel Gear

position of joint after link elasticity and joint coupling. Accordingly, $\boldsymbol{\tau}_{\boldsymbol{l}}$ and $\boldsymbol{\tau}_{\boldsymbol{q}}$ represent joint torques in joint and motor coordinates, while $\boldsymbol{\tau}_{\boldsymbol{q}}$ will be denoted as $\tau$ later in this paper.

Experimental results have shown that the elastic deformation of the joints, either through deformation of the bearing or of the gear teeth, would also introduce joint flexibility. Therefore the joint elasticity is taken into account in the modeling of robotic manipulators in this paper, which is modeled by a linear torsional spring with stiffness $\boldsymbol{K}$. The kinematics model of the robot hand with elastic joint and coupled joints can then be expressed as below [15]:

$$
\begin{gathered}
M(q) \ddot{q}+C(q, \dot{q}) \dot{q}+g(q)=\tau+\tau_{e x t} \\
J_{m} \ddot{\theta}_{m}+L^{-T} \tau+\tau_{f, m}=\tau_{m} \\
\tau=K\left(L q-\theta_{m}\right)
\end{gathered}
$$

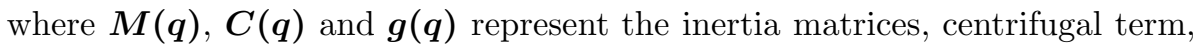
and gravity term, respectively. The joint torque vector is given by $\boldsymbol{K}\left(\boldsymbol{L} \boldsymbol{q}-\boldsymbol{\theta}_{\boldsymbol{m}}\right)$, where $\boldsymbol{\theta}_{\boldsymbol{m}}$ indicates the vector of the motor angle divided by the gear ratio, and $\boldsymbol{q}$ represents the link side joint angle. $\boldsymbol{K}, \boldsymbol{J}_{\boldsymbol{m}}$ are diagonal matrices which contain the joint stiffness, and the motor inertia multiplied by the gear ratio squared. $\tau_{\boldsymbol{e x t}}$ and $\boldsymbol{\tau}_{\boldsymbol{f}, \boldsymbol{m}}$ are external torque vector and friction torque vector, respectively. The generalized actuator torque vector, $\boldsymbol{\tau}_{\boldsymbol{m}}$, is considered as the control input.

\section{Impedance Controller Design for Dexterous Robot Hand}

The goal of the impedance controller is to achieve a desired dynamic behavior with respect to external forces and torques acting on the link side of the end 
effector. As described in last section, robot hand dynamics can be expressed in the joint coordinates system by using a transformation matrix $\boldsymbol{L}$. Since elasticity in the joints of DLR-HIT II robot hand is not negligible, resulting dynamics of a motor position-based PD controller is influenced by the joint elasticity and the motor inertia. By a negative feedback of the joint torque $\boldsymbol{\tau}$, the apparent inertia can be scaled down such that the closed-loop system reacts to external forces $\boldsymbol{F}_{\text {ext }}$ as if the robot inertia were smaller [18, which can be represented as:

$$
\tau_{j}=J J_{c}^{-1} u+\left(I-J J_{c}^{-1}\right) \tau
$$

with

$$
\begin{aligned}
\tau_{j} & =L^{T}\left(\tau_{m}-\tau_{f, m}\right) \\
J & =L^{T} J_{m} L
\end{aligned}
$$

where $\boldsymbol{u} \in \mathbb{R}$ is the new input variable, and $\boldsymbol{J}_{\boldsymbol{c}}$ is a diagonal, positive define matrix with $\boldsymbol{j}_{\boldsymbol{c} i}<\boldsymbol{j}_{\boldsymbol{i}}$.

The position and orientation of the end-effector can be described by a set of local coordinates $\boldsymbol{x} \in \mathbb{R}^{\boldsymbol{m}}$, and the relationship between Cartesian coordinates $\boldsymbol{x}$ and the configuration coordinates $\boldsymbol{q} \in \mathcal{Q}$ is given by a known function $\boldsymbol{f}$ : $\mathcal{Q} \rightarrow \mathbb{R}^{\boldsymbol{m}}$, i.e. $\boldsymbol{x}=\boldsymbol{f}(\boldsymbol{q})$. With the Jacobian $\boldsymbol{J}(\boldsymbol{q})=\boldsymbol{\partial} \boldsymbol{f}(\boldsymbol{q}) / \boldsymbol{\partial q}$, Cartesian velocities and accelerations can be expressed as

$$
\begin{gathered}
\dot{x}=J(q) \dot{q} \\
\ddot{x}=J(q) \ddot{q}+\dot{J}(q) \dot{q}
\end{gathered}
$$

Throughout this paper only the nonsingular case is considered, thus it is assumed that the manipulator's Jacobian $\boldsymbol{J}(\boldsymbol{q})$ has full row rank in the considered region of the workspace.

To specify the desired impedance behavior, the position error $\tilde{\boldsymbol{x}}=\boldsymbol{x}-\boldsymbol{x}_{\boldsymbol{d}}$, between real position $\boldsymbol{x}$ and a virtual equilibrium position (possibly time-varying) $\boldsymbol{x}_{\boldsymbol{d}}$, is introduced. The goal of the impedance controller design here is to alter the system dynamics (4) such that, in the presence of external forces and torques at the end-effector $\boldsymbol{F}_{\boldsymbol{e x t}} \in \mathbb{R}^{\boldsymbol{m}}$, a dynamic relationship between $\tilde{\boldsymbol{x}}$ and $\boldsymbol{F}_{\boldsymbol{e x t}}$ could be achieved as follows:

$$
\Lambda_{d} \ddot{\tilde{x}}+D_{d} \dot{\tilde{x}}+K_{d} \tilde{x}=F_{e x t}
$$

where $\boldsymbol{\Lambda}_{\boldsymbol{d}}, \boldsymbol{D}_{\boldsymbol{d}}$ and $\boldsymbol{K}_{\boldsymbol{d}}$ are the symmetric and positive definite matrices of the desired inertia, damping and stiffness, respectively.

The relationship between the external torque vector $\tau_{\boldsymbol{e x t}}$ and the generalized external force vector $\boldsymbol{F}_{\boldsymbol{e x t}}$ on the end-effector is given by:

$$
\tau_{\text {ext }}=J(q)^{T} F_{\text {ext }}
$$

By substituting (10), (11) and (13) into (4) and rewriting $\boldsymbol{g}(\boldsymbol{q}), \boldsymbol{\tau}$ in the form of the equivalent task space as $\boldsymbol{F}_{\boldsymbol{g}}(\boldsymbol{x}), \boldsymbol{F}_{\boldsymbol{\tau}}$, the relationship between the Cartesian coordinates $\boldsymbol{x}$ and the joint torques $\boldsymbol{\tau}$ can be expressed as:

$$
\Lambda(x) \ddot{x}+\mu(x, \dot{x}) \dot{x}+F_{g}(x)=F_{\tau}+F_{e x t}
$$


where $\boldsymbol{\Lambda}(\boldsymbol{x})$ and $\boldsymbol{\mu}(\boldsymbol{x}, \dot{\boldsymbol{x}})$ are the inertia matrix and the Coriolis/centrifugal matrix with respect to the coordinates $\boldsymbol{x}$, and given by:

$$
\begin{aligned}
\Lambda(x) & =J(q)^{-T} M(q) J(q)^{-1} \\
\mu(x, \dot{x}) & =J(q)^{-T}\left(C(q, \dot{q})-M(q) J(q)^{-1} \dot{J}(q)\right) J(q)^{-1}
\end{aligned}
$$

with $\boldsymbol{q}=\boldsymbol{f}^{-1}(\boldsymbol{x})$ and $\dot{\boldsymbol{q}}=\boldsymbol{J}\left(\boldsymbol{f}^{-1}(\boldsymbol{x})\right) \dot{\boldsymbol{x}}$.

Combining (14) and (12), the impedance control law, which is the desired closed loop system, with $\boldsymbol{F}_{\boldsymbol{\tau}}$ as the control input, can be arrived:

$$
\begin{aligned}
F_{\tau}= & \Lambda(x) \ddot{x}_{d}+\mu(x, \dot{x}) \dot{x}+\left(\Lambda(x) \Lambda_{d}^{-1}-I\right) F_{e x t} \\
& +F_{g}(x)-\Lambda(x) \Lambda_{d}^{-1}\left(D_{d} \dot{\tilde{x}}+K_{d} \tilde{x}\right)
\end{aligned}
$$

If the desired torque vector $\tau$ is chosen as:

$$
\tau=J(q)^{T} F_{\tau}+C(q, \dot{q}) \dot{q}-J(q)^{T} \Lambda(x) \dot{J}(q) J(q)^{-1}
$$

With the assumption that centripetal and Coriolis forces can be ignored at the robot's relatively low operating speeds. Furthermore, If the desired intertia $\boldsymbol{\Lambda}_{\boldsymbol{d}}$ is chosen as identical to the robot inertia $\boldsymbol{\Lambda}(\boldsymbol{x})$, the feedback of external forces $\boldsymbol{F}_{\boldsymbol{e x t}}$ can be avoided. This results in the following actual implementation of the impedance controller:

$$
F_{\tau}=\Lambda(x) \ddot{x}_{d}-D_{d} \dot{\tilde{x}}-K_{d} \tilde{x}+F_{g}(x)
$$

and the desired joint torques $\tau$ :

$$
\tau=g(q)+J(q)^{T}\left(\Lambda(x) \ddot{x}_{d}-D_{d} \dot{\tilde{x}}-K_{d} \tilde{x}\right)
$$

Using motor $\boldsymbol{\theta}$ instead of the link side angles $\boldsymbol{q}$ in the forward kinematics $\boldsymbol{x}=\boldsymbol{f}(\boldsymbol{q})$, impedance controller based on PD position control in the Cartesian coordinates can be generalized. The feedback law is given by:

$$
\begin{aligned}
u & =-J(\theta)^{T}\left(K_{x} \tilde{x}(\theta)+D_{x} \dot{x}\right) \\
\tilde{x}(\theta) & =f(\theta)-x_{d} \\
\dot{x} & =J(\theta) \dot{\theta}
\end{aligned}
$$

with $\boldsymbol{K}_{\boldsymbol{x}}$ and $\boldsymbol{D}_{\boldsymbol{x}}$ represent the desired stiffness and damping matrices, respectively corresponding to $\boldsymbol{K}_{\boldsymbol{d}}$ and $\boldsymbol{D}_{\boldsymbol{d}}$ in (21). $\boldsymbol{x}_{\boldsymbol{d}}$ indicates the virtual motor side position in Cartesian coordinates. As such, the controller in (22), together with (7), forms the closed loop system:

$$
\begin{array}{r}
M(q) \ddot{q}+C(q, \dot{q})+g(q)=\tau+\tau_{\text {ext }} \\
J_{c} \ddot{\theta}+J(\theta)^{T}\left(K_{x} \tilde{x}(\theta)+D_{x} \dot{x}\right)+\tau=\bar{g}(\theta)
\end{array}
$$

Since link side deviations from the steady state positions due to gravity is not negligible, the following Lyapunov function $\boldsymbol{V}(\boldsymbol{q}, \dot{\boldsymbol{q}}, \boldsymbol{\theta}, \dot{\boldsymbol{\theta}})$ is chosen:

$$
\begin{aligned}
V(q, \dot{q}, \theta, \dot{\theta})= & \frac{1}{2} \dot{q}^{T} M(q) \dot{q}+\frac{1}{2} \dot{\theta}^{T} J_{c} \dot{\theta}+\frac{1}{2}(\theta-q)^{T} K(\theta-q) \\
& +\frac{1}{2} \tilde{x}(\theta)^{T} K_{x} \tilde{x}(\theta)+V_{g}(q)-V_{\bar{g}}(\theta) \\
\geq & 0
\end{aligned}
$$


For $\boldsymbol{\tau}_{\boldsymbol{e x t}}=\mathbf{0}$, derivative of Lyapunov function can be manipulated:

$$
V(q, \dot{q}, \theta, \dot{\theta})=-\dot{x}^{T} D_{x} \dot{x} \leq 0
$$

The system converges to invariant set contained in subspace $\boldsymbol{P}=\left(\boldsymbol{\theta}_{\boldsymbol{s}}, \mathbf{0}, \boldsymbol{q}_{\boldsymbol{s}}, \mathbf{0}\right)^{\boldsymbol{T}}$, which is actually the steady state given by (23):

$$
\begin{aligned}
K\left(\theta_{s}-q_{s}\right) & =g\left(q_{s}\right) \\
K\left(\theta_{s}-q_{s}\right)+J\left(\theta_{s}\right)^{T} K_{x} \tilde{x}\left(\theta_{s}\right) & =\bar{g}(\theta)
\end{aligned}
$$

Then the asymptotic stability is shown by the LaSalle Invariance Principle.

\section{Velocity Observer and Friction Compensation}

\subsection{Friction Model Linearization and Parameters Estimation}

Performance of the proposed impedance controller can be hindered by nonlinear friction. In this paper, friction model $\tau_{f, m}$ is composed of Coulomb friction $\tau_{c}$ and viscous friction $\boldsymbol{\tau}_{\boldsymbol{v}}$, which can be written as:

$$
\tau_{f, m}=\tau_{c}+\tau_{v}=\alpha \operatorname{sign}\left(\dot{\theta}_{m}\right)+\beta \dot{\theta}_{m}
$$

with $\boldsymbol{\alpha}$ and $\boldsymbol{\beta}$ denoting the Coulomb and viscous coefficient, respectively. The following differential equation represents the resulting dynamics of the system with the friction model:

$$
J_{c} \ddot{\theta}+\alpha \operatorname{sign}\left(\dot{\theta}_{m}\right)+\beta \dot{\theta}_{m}=\tau_{m}
$$

In order to implement a controller with friction compensation, it is necessary to determine parameters corresponding to the robot dynamic model. Nonlinearity of the above friction model resides in the signum function term $\operatorname{sign}(\dot{\boldsymbol{\theta}})$. By multiplying both sides of the equation (29) with term $\dot{\boldsymbol{\theta}}$, the friction model is given by linear form:

$$
J_{c} \dot{\theta} \ddot{\theta}+\beta \dot{\theta}^{2}+\alpha \underbrace{\dot{\theta} \operatorname{sign}(\dot{\theta})}_{|\dot{\theta}|}=\dot{\theta} \tau
$$

By integrating (30) over one timestep $\boldsymbol{t}_{\boldsymbol{s}}=\boldsymbol{t}_{\boldsymbol{k}+\mathbf{1}}-\boldsymbol{t}_{\boldsymbol{k}}$ with a trapzoidal approximation, the least-squares estimation can be achieved:

$$
\underbrace{\left[\begin{array}{c}
\frac{1}{2}\left(\dot{\theta}_{k+1}^{2}-\dot{\theta}_{k}^{2}\right) \frac{t_{s}}{2}\left(\dot{\theta}_{k+1}^{2}+\dot{\theta}_{k}^{2}\right) \\
\downarrow k=0 \ldots N \\
\vdots
\end{array}\right]}_{L S}\left[\begin{array}{c}
t_{s}\left(\left|\dot{\theta}_{k+1}^{2}\right|+\left|\dot{\theta}_{k}^{2}\right|\right) \\
\beta \\
\alpha
\end{array}\right]=\left[\begin{array}{c}
\frac{t_{s}}{2}\left(\tau_{k+1} \dot{\theta}_{k+1}-\tau_{k} \dot{\theta}_{k}\right) \\
\downarrow k=0 \ldots N \\
\vdots
\end{array}\right]
$$




\subsection{Velocity Observer and Friction Estimation}

The extended Kalman filter (EFK) is adopted in this paper to estimate velocity and friction, which is subdivided into prediction step and estimation step as shown in 21. Based on the current state and the dynamic model of the system, a forecast can be calculated for the state in the prediction step:

$$
\begin{aligned}
\Delta \hat{x}_{k+1}^{-} & =A_{k} \Delta \hat{x}_{k}^{+}+B_{K} u_{k} \\
P_{k+1}^{-} & =A_{k} P_{k}^{+}+G_{K} Q_{k} G_{k}^{T}
\end{aligned}
$$

The estimation step is defined, where the forecast and the measurements are compared, to arrive the following:

$$
\begin{aligned}
K_{k} & =P_{k}^{-} H_{k}^{T}\left(H_{k} P_{k}^{-} H_{k}^{T}+R_{k}\right)^{-1} \\
\Delta \hat{x}_{k}^{+} & =\Delta \hat{x}_{k}^{-}-K\left(H_{k} \Delta \hat{x}_{k}^{-}-\Delta y_{k}\right) \\
P_{k}^{+} & =\left(I-K_{k} H_{k}\right) P_{k}^{-}
\end{aligned}
$$

In order to eliminate a possible divergence of $\boldsymbol{\Delta} \boldsymbol{x}$, the error state is set to zero after the estimation step.

To derive an extended Kalman filter, viscous and static friction parameters $\boldsymbol{b}$ and $\boldsymbol{c}$ are modeled as constant system states. $\boldsymbol{\tau}$ is considered as a state variable, or specifically a measurement variable rather than an input variable. The dynamic model of the system can be expressed as [22]:

$$
\frac{d}{d t} \underbrace{\left(\begin{array}{c}
\theta \\
\dot{\theta} \\
\tau \\
b \\
c
\end{array}\right)}_{:=x}=\underbrace{\left(\begin{array}{c}
\dot{\theta} \\
\frac{1}{J_{C}}(\tau-b \dot{\theta}-c \operatorname{sign}(\dot{\theta})) \\
0 \\
0 \\
0
\end{array}\right)}_{:=a}
$$

By partial derivation of the system dynamics equation and replacing the partial derivation sign $\boldsymbol{\partial}$ with differences sign $\boldsymbol{\Delta}$. The following linearized system dynamics equation can be achieved:

$$
\frac{d}{d t}\left(\begin{array}{c}
\Delta \theta \\
\Delta \dot{\theta} \\
\Delta \tau \\
\Delta b \\
\Delta c
\end{array}\right)=A_{l i n} \cdot\left(\begin{array}{c}
\Delta \theta \\
\Delta \dot{\theta} \\
\Delta \tau \\
\Delta b \\
\Delta c
\end{array}\right)
$$


where:

$$
\begin{aligned}
& A_{l i n}=\left(\begin{array}{lllll}
0 & 1 & 0 & 0 & 0 \\
0 & -\left(\frac{b}{J_{c}}+\alpha \frac{c}{J_{c}}\right) & \frac{1}{J_{c}} & \frac{-\dot{\theta}}{J_{c}} & \frac{-f(\dot{\theta})}{J_{c}} \\
0 & 0 & 1 & 0 & 0 \\
0 & 0 & 0 & 1 & 0 \\
0 & 0 & 0 & 0 & 1
\end{array}\right) \\
& f(\dot{\theta})=\left\{\begin{array}{ccc}
-1 & \dot{\theta}<- \text { limit } \\
\frac{1}{\text { limit }} & - \text { limit }<\dot{\theta}<\text { limit } \\
1 & \text { limit }<\dot{\theta}
\end{array}\right.
\end{aligned}
$$

with $\boldsymbol{\alpha}$ represents the derivation of $\boldsymbol{f}(\dot{\boldsymbol{\theta}})$, given by:

$$
\alpha=\left\{\begin{array}{cc}
0 & \dot{\theta}<- \text { limit } \\
\frac{1}{\text { limit }} & - \text { limit }<\dot{\theta}<\text { limit } \\
\mathbf{0} & \text { limit }<\dot{\theta}
\end{array}\right.
$$

Considering that $\boldsymbol{\alpha}$ and $\boldsymbol{\beta}$ are constant and estimated with least squares in the prior section, the linearized extended Kalman filter can be achieved by solving (34):

$$
\Delta x_{k+1}=\left(\begin{array}{ccc}
1 & a_{2} & a_{3} \\
0 & a_{1} & \frac{1}{J_{c}} \cdot a_{2} \\
0 & 0 & 1
\end{array}\right) \cdot \Delta x_{k}
$$

Where:

$$
\begin{aligned}
& a_{1}=e^{-(b+\alpha c) \cdot \frac{t_{s}}{J_{c}}} \\
& a_{2}=\frac{J_{c}}{b+\alpha c} \cdot\left(1-e^{-(b+\alpha c) \cdot \frac{t_{s}}{J_{c}}}\right) \\
& a_{3}=\frac{J_{c}}{(b+\alpha c)^{2}} \cdot\left(e^{-(b+\alpha c) \cdot \frac{t_{s}}{J_{c}}}-1+(b+\alpha c) \frac{t_{s}}{J_{c}}\right)
\end{aligned}
$$

\section{Experiments}

The Cartesian impedance control experiment is carried out in the five-finger dexterous robot hand DLR-HIT II with a hard real time control cycle $200 \boldsymbol{\mu s}$. Multi-point LVDS serial communication system and QNX real time OS are utilized to fulfill the requirements of the proposed controller. $\boldsymbol{D}_{\boldsymbol{x}}$ and $\boldsymbol{K}_{\boldsymbol{k}}$ are designed by the double-diagonalization approach with the robot inertia matrix and the desired damping ratio, as presented in [23. Other parameters needed for implementing the impedance controller (23) can be generated by directly using Pro/E model of the dexterous hand.

In the following two experiments, the former one is conducted to test the performance of the controller, whereas the latter one is carried out to show the compliant behavior of the robot hand. The designed Cartesian impedance controller is implemented in a single finger of the robot hand. The finger tracks 
the desired position trajectory(red line) and makes contact with a rigid external object at the position offset $\boldsymbol{\Delta} \boldsymbol{x}=\mathbf{0 . 0 1 1} \boldsymbol{m}$ in the $\boldsymbol{x}$ direction, as shown in Fig. 3. Together with Cartesian force response in Fig. 3, the experimental results show that the proposed impedance controller is effective in position tracking.
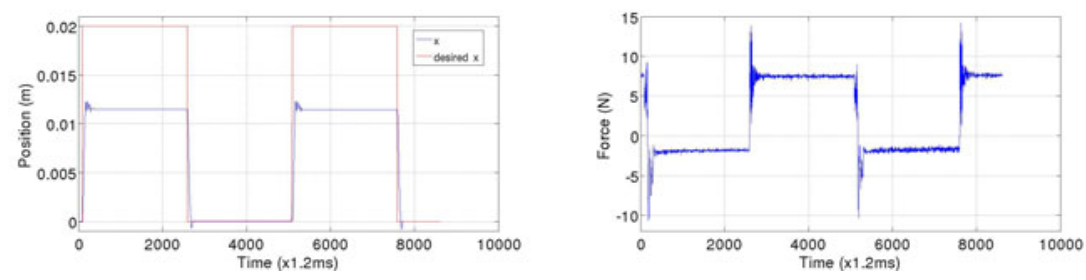

Fig. 3. Position tracking and force response

As shown in Fig. 4. The robot overcomes the gravity and friction, returning to the equilibrium Cartesian position $\boldsymbol{x}_{\boldsymbol{d}}$ as soon as the external force is released. With the friction and gravity compensation proposed in this paper, the static error in the $\boldsymbol{x}$ axis is less than $0.2 \mathrm{~mm}$, as well as $\boldsymbol{y}$ and $\boldsymbol{z}$ direction. It can therefore be concluded that the Cartesian impedance controller is successfully realized.
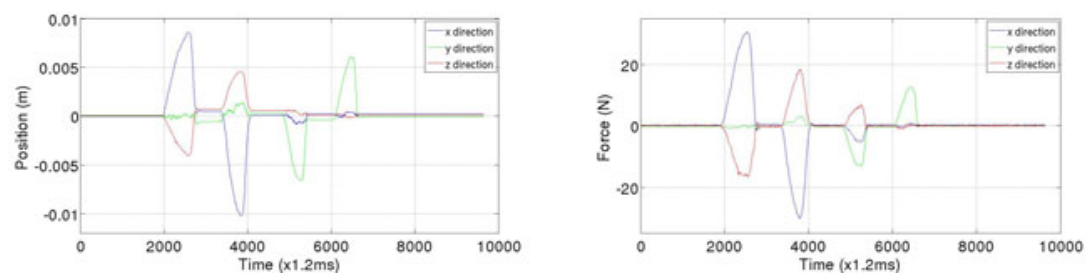

Fig. 4. Cartesian coordinate position and force response with impedance controller

\section{Conclusions}

In this paper, a Cartesian impedance controller with friction compensation is derived for the dexterous robot hand DLR-HIT II hand with flexible joints. Global asymptotic stability is guaranteed by LaSalle Principle analysis. To improve the performance of the proposed controller, model based friction compensation is adopted in this paper, in which friction parameters are estimated with the Least Squares Method. Together with joint velocity observed by extended Kalman filter, non-linear friction compensation can be derived. Two experiments are carried out on the DLR-HIT hand to show the effectiveness of designed impedance controller with friction compensation and its compliant behavior with the robot hand. For the harmonic drive robot hand with joint torque feedback, accurate position tracking and stable torque/force response can be achieved with the proposed Cartesian impedance controller. 


\section{References}

1. Bicchi, A.: Hands for dexterous manipulation and robust grasping: a difficultroad toward simplicity. IEEE Transactions on Robotics and Automation 16(6), 652-662 (2000)

2. Salisbury, J., Craig, J.: Articulated hands: Force control and kinematic issues. The International Journal of Robotics Research 1(1), 4 (1982)

3. Jacoben, S., et al.: The Utah/MIT dexterous hand: Work in progress. Int. J. Robot 3(4), 21-50 (1984)

4. Lovchik, C., Difler, M.: The robonaut hand: A dextrous robotic hand for space. In: Proceedings of the IEEE International Conference on Robotics and Automation, pp. 907-912 (1999)

5. Kawasaki, H., Komatsu, T., Uchiyama, K.: Dexterous anthropomorphic robot hand with distributed tactile sensor: Gifu hand II, IEEE. ASME Trans. on Mechatronics 7(3), 296-303 (2002)

6. Butterfass, J., Grebenstein, M., Liu, H.: DLR-Hand II: Next generation of a dexterous robot hand. In: Proceedings of the 2001 IEEE International Conference on Robotics \& Automation, pp. 109-114 (2001)

7. Liu, H., Meusel, P., Hirzinger, G., Jin, M., Liu, Y., Xie, Z.: The modular multisensory DLR-HIT-hand: Hardware and software architecture. IEEE/ASME Transactions on Mechatronics 13(4), 461-469 (2008)

8. Chiaverini, S., Sciavicco, L.: The parallel approach to force/position control of roboticmanipulators. IEEE Transactions on Robotics and Automation 9(4), 361373 (1993)

9. Volpe, R., Khosla, P.: A theoretical and experimental investigation of explicit forcecontrol strategies for manipulators. IEEE Transactions on Automatic Control 38(11), 1634-1650 (1993)

10. Roy, J., Whitcomb, L.: Adaptive force control of position/velocity controlled robots: theory and experiment. IEEE Transactions on Robotics and Automation 18(2), 121-137 (2002)

11. Hogan, N.: Impedance control-An approach to manipulation. I-Theory. IIImplementation. III-Applications. ASME, Transactions, Journal of Dynamic Systems, Measurement and Control 107 (1985), ISSN: 0022-0434

12. Kazerooni, H., Houpt, P., Sheridan, T.: Robust compliant motion for manipulators: the fundamental concepts of compliant motion (part I); design method (part II). IEEE Journal of Robotics and Automation (legacy, pre-1988) 2(2), 83-105 (1986)

13. Khatib, O.: A unified approach for motion and force control of robot manipulators: The operational space formulation. IEEE Journal of Robotics and Automation 3(1), 43-53 (1987)

14. Bonitz, R., Hsia, T.: Internal force-based impedance control for cooperating manipulators. IEEE Transactions on Robotics and Automation 12(1), 78-89 (1996)

15. Spong, M., Khorasani, K., Kokotovic, P.: An integral manifold approach to the feedback control of flexible joint robots. IEEE Journal of Robotics and Automation (legacy, pre-1988) 3(4), 291-300 (1987)

16. Tian, L., Goldenberg, A.: Robust adaptive control of flexible joint robots with joint torque feedback. In: Proceedings of 1995 IEEE International Conference on Robotics and Automation, vol. 1 (1995)

17. De Luca, A., Lucibello, P.: A general algorithm for dynamic feedback linearization of robots with elastic joints. In: IEEE International Conference on Robotics and Automation, pp. 504-510 (1998) 
18. Ott, C., Albu-Schaeffer, A., Kugi, A., Hirzinger, G.: On the Passivity-Based Impedance Control of Flexible Joint Robots. IEEE Transactions on Robotics 24(2), 416-429 (2008)

19. Albu-Schaeffer, A., Ott, C., Hirzinger, G.: A unified passivity-based control framework for position, torque and impedance control of flexible joint robots. The International Journal of Robotics Research 26(1), 5-21 (2007)

20. Liu, H., Wu, K., Meusel, P., Seitz, N., Hirzinger, G., Jin, M., Liu, Y., Fan, S., Lan, T., Chen, Z.: Multisensory five-finger dexterous hand: The DLR/HIT Hand II. In: IEEE/RSJ International Conference on Intelligent Robots and Systems, IROS 2008, pp. 3692-3697 (2008)

21. Gelb, A.: Applied optimal estimation. MIT Press, Cambridge (2002)

22. Connette, C.P.: Intern Report On DLR Hand II, Institute of Robotic and Mechatronics, DLR (2006)

23. Albu-Schaeffer, A., Ott, C., Hirzinger, G.: A passivity based Cartesian impedance controller for flexible joint robots-Part II: full state feedback, impedance design and experiments. In: IEEE International Conference on Robotics and Automation, Citeseer, pp. 2666-2672 (2004) 